\title{
B Research S Suare \\ Potential Biological Molecules and Immune Cell for Rheumatoid Arthritis Synovial Lesions
}

\author{
Mingyi Yang \\ Xi'an Red Cross Hospital \\ Yani Su \\ Yan'an University \\ Yao Ma \\ Xi'an Red Cross Hospital \\ $\mathrm{KeXu}$ \\ Xi'an Red Cross Hospital \\ Aihaiti Yirixiati \\ Xi'an Red Cross Hospital \\ Peng Xu ( $\sim$ sousou369@163.com ) \\ Xi'an Red Cross Hospital https://orcid.org/0000-0003-2487-9163
}

\section{Research article}

Keywords: rheumatoid arthritis, genes, pathways, miRNAs, immune cells

Posted Date: May 10th, 2021

DOl: https://doi.org/10.21203/rs.3.rs-491259/v1

License: (c) (1) This work is licensed under a Creative Commons Attribution 4.0 International License.

Read Full License 


\section{Abstract}

Objective: To study the potential biomarkers and related pathways in rheumatoid arthritis (RA) synovial lesions, as well as immune cell, to provide theoretical basis and research directions for the mechanism and treatment of RA. Methods: Download the RA synovial tissue microarray data set (GSE77298, GSE55457 and GSE55235) from Gene Expression Omnibus (GEO), The"limma" package of R to identify differentially expressed genes (DEGs), DAVIA Perform GO (Gene Ontology, gene ontology) and KEGG (Kyoto Encyclopedia of Genes and Genomes, Kyoto Gene Encyclopedia) enrichment analysis. STRING (Search Tool for the Retrieval of Interacting Genes) constructs a Protein Protein Interaction Network (PPI), R screens Hub genes, and mines targeted miRNAs of Hub genes based on multiple databases. In R,the immune cell of RA synovial tissue samples are obtained through the three packages of "e1071", "parallel" and "preprocessCore" with "CIBERSORT" software. Results: Ten Hub genes (KIAA0101, FOXM1, EGFR, CDC20, BUB1B, TYMS, TOP2A, RRM2, JUN and CCNA2) and 2 key miRNAs (miR-520d-5p and miR) related to RA synovial lesions were finally identified -139-5p), significantly enriched in epithelial cell signaling, ECM-receptor interaction, estrogen signaling pathway, cell cycle, ErbB signaling pathway and $\mathrm{GnRH}$ signaling pathway in Helicobacter pylori infection. Immune cell analysis found that resting dendritic cells, $B$ cells memory, dendritic cells activated, plasma cells, macrophages $M 1$, mast cells resting and $T$ cells regulatory have high expression in RA, while neutrophils, $B$ cells naive and natural killer cells activated have low expression in RA. Conclusion:The Hub genes, key miRNAs, related pathways, and immune cell obtained in this study provide a certain basis for the etiology and treatment of RA synovial lesions.

\section{Introduction}

Rheumatoid arthritis (RA) is a chronic autoimmune disease of unknown etiology, mainly polyarticular disease. Its pathological changes are the proliferation of synovial cells, release of inflammatory factors and deposits in joints and cartilage, causing joint swelling and pain , Deformity, and loss of function, characterized by excessive activation of collagen-specific T helper cells and increased levels of autoantibodies in serum (1). Because of its long course, the disease is progressive, and the late stage often leads to joint deformities and dysfunction. It is the most obvious and most commonly disabling arthritis. RA affects about $1 \%$ of the global population, women are more common than men, and the incidence is highest in patients between 40 and 60 years old (2). The disease is most typical in women and the elderly. Uncontrolled active RA can lead to joint damage, disability, reduced quality of life, cardiovascular and other diseases. Relieving synovitis and systemic inflammation, improving function is the key to treating rheumatism (3). So far there is no complete cure for RA, so some people also call RA "cancer that can't die." The cure of RA has become the demand of the whole society, but the pathogenesis of the disease is still unclear. In recent years, microarray technology and bioinformatics analysis have been widely used to screen potential biomarkers of diseases, providing a certain basis for the study of disease molecular mechanisms (4). This study analyzed the Hub gene and its related pathways, key 
miRNAs and immune cell in RA synovial lesions through bioinformatics, and provided a new theoretical basis for the etiology and treatment of RA.

\section{Materials And Methods}

\section{Microarray data}

GEO (http://www.ncbi.nlm.nih.gov/geo) is part of the NCBI of the National Institutes of Health, and is the largest and most comprehensive public data resource today. Download three gene expression datasets GSE77298, GSE55457 and GSE55235 from GEO. The GSE77298 dataset contains 16 RA synovial tissue samples and 7 normal synovial tissue samples. The GSE55457 dataset contains 13 RA synovial tissue samples and 10 normal synovial tissue samples. The GSE55235 dataset contains 10 RA Synovial tissue samples and 10 normal synovial tissue samples.

\section{Identify DEGs}

$\mathrm{R}$ is a language and operating environment for statistical analysis and drawing. It is a free, open source software and an excellent tool for statistical calculation and statistical drawing. Perl is another programming language that can accomplish tasks. Perl, like a scripting language, does not require an editor and linker to run the code, just write the program and tell Perl to run it. The three datasets of GSE77298, GSE55457 and GSE55235 were combined with Perl, R was used for batch calibration, and the "limma" package of $R$ was used to identify DEGs. The screening criteria were: $P<0.05$, $\log \mathrm{FC} \geq 1 / \log \mathrm{FC} \leq-1$. Further visualizes the volcano map and heat map.

\section{GO and KEGG enrichment analysis of DEGs}

DAVID (http://david.ncifcrf.gov, version 6.8) is a biological information database that integrates biological data and analysis tools. It provides systematic and comprehensive biological function annotation information for large-scale gene or protein lists to help users Extract biological information from it(5). GO is a major bioinformatics tool for annotating genes and analyzing the biological processes of these genes (6). KEGG is a database that integrates genome, chemistry and system function information, and is a computer simulation of a biological system. DAVID performed GO and KEGG enrichment analysis on DEGs, and the selection criterion was P-value $<0.05$.

\section{Construction of PPI network and identification of Hub gene}

STRING(http://string-db.org) (version 11.0) is an online database for searching known protein interaction relationships. It can predict the interaction gene network and provide a deeper understanding of the mechanism of disease generation or development. Scientific basis. Using STRING to construct the PPI network of DEGs, the interaction with a comprehensive score $>0.4$ was considered statistically significant. $\mathrm{R}$ identifies the Hub gene with the highest network connectivity in the PPI network. 
TargetScan, miRDB and miRWalk three databases predict the target miRNAs of Hub gene, and the prediction results are intersected. Cytoscape constructs a Hub gene-miRNAs interaction network, and miRNAs targeting two or more Hub genes are considered key miRNAs (7).

\section{Immune cell infiltration of RA synovial tissue}

The three data sets and the corrected expression matrix are used in R through the three packages "e1071", "parallel", and "preprocessCore" to obtain the immune cell matrix of RA synovial tissue samples using "CIBERSORT" software. Perl screens the samples with $P<0.05$ in the immune cell matrix and visualizes them with $\mathrm{R}$.

\section{Results}

\section{Identification of DEGs}

After the three datasets of GSE77298, GSE55457 and GSE55235 were merged, there were 39 RA synovial tissue samples and 27 normal synovial tissue samples. R performs batch calibration (Figure 1). The "limma" package identified 84 DEGs, including 43 upregulated DEGs and 44 downregulated DEGs. R visualizes volcano maps and heatmap on DEGs (Figure 2).

\section{GO and KEGG enrichment analysis of DEGs}

DAVID performed GO and KEGG enrichment analysis on DEGs. Biological pathways (BP) were significantly enriched in immune response (GO: 0006955), positive regulation of cell migration (GO: 0030335), negative regulation of apoptotic process (GO : 0043066) and positive regulation of cell proliferation (GO: 0008284). Cellular component (CC) is significantly enriched in basement membrane (GO: 0005604) and extracellular area (GO: 0005576). Molecular function (MF) is significantly enriched in enzyme binding (GO: 0019899) and sequence-specific DNA binding (GO: 0043565) (Figure 3A). KEGG was significantly enriched in Epithelial cell signaling in Helicobacter pylori infection, ECM-receptor interaction, estrogen signaling pathway, cell cycle, ErbB signaling pathway, and GnRH signaling pathway (Figure 3B). The pathway diagram of GnRH signaling pathway is shown in (Figure 4).

\section{Construction of PPI network and identification of Hub gene}

STRING builds a PPI network of DEGs with 86 nodes and 269 edges. R identified Hub genes, and a total of 10 Hub genes were identified, namely KIAA0101, FOXM1, EGFR, CDC20, BUB1B, TYMS, TOP2A, RRM2, JUN and CCNA2 (Figure 5). The detailed information of Hub gene is shown in (Table 1).

\section{Hub gene-miRNAs interaction network analysis and key miRNAs prediction}

TargetScan, miRDB and miRWalk predict the target miRNAs of Hub gene, and Cytoscape constructs the interaction network of Hub gene and miRNAs (Figure 6). The miRNAs targeting two or more Hub genes at the same time are defined as key miRNAs, and a total of 2 key miRNAs (miR-520d-5p and miR-139-5p) 
are obtained. miR-520d-5p corresponds to the three genes BUB1B, TOP2A and JUN, and miR-139-5p corresponds to the two genes TOP2A and JUN.

\section{Immune cell infiltration of RA synovial tissue}

The three datasets and the corrected expression matrix yielded a total of 22 immune cell expression matrices, including 66 samples (39 RA synovial tissue samples and 27 normal synovial tissue samples). After screening with $P<0.05,13$ samples (10 RA synovial tissue samples and 3 normal synovial tissue samples) were obtained. $R$ visualization histogram (Figure 7), the "pheatmap" package of $R$ visualization heatmap (Figure 8). We found that resting dendritic cells, B cells memory, dendritic cells activated, plasma cells, macrophages $\mathrm{M} 1$, mast cells resting and T cells regulatory have high expression in RA, while neutrophils and B cells naive have low expression in RA. "Vioplot" package of R were used to further visualize the violin chart of the selected immune cell matrix, we found that macrophages M1 ( $P=0.049)$ have high expression in $R A, B$ cells naive $(P=0.009)$ and natural killer cells activated $(P=0.028)$ have low expression in RA (Figure 9).

\section{Discussion}

RA leads to progressive joint destruction, disability and increased mortality, which seriously affects the health of patients, but its pathogenesis is still unclear. Bioinformatics provides a powerful method for the identification of disease biomarkers and related methods, and further promotes the research of disease etiology and pathogenesis. We conducted bioinformatics analysis on RA synovial tissue and finally identified 10 Hub genes (KIAA0101, FOXM1, EGFR, CDC20, BUB1B, TYMS, TOP2A, RRM2, JUN and CCNA2) and two key miRNAs (miR-520d-5p and miR-139-5p) related to RA synovial tissue. Pathway enrichment is significantly enriched in Epithelial cell signaling in Helicobacter pylori infection, ECMreceptor interaction, estrogen signaling pathway, cell cycle, ErbB signaling pathway, and GnRH signaling pathway. Immune cell analysis found that resting dendritic cells, B cells memory, dendritic cells activated, plasma cells, macrophages $M 1$, mast cells resting and T cells regulatory have high expression in RA, while neutrophils, B cells naive and natural killer cells activated have low expression in RA.

The KIAA0101 gene encodes a proliferating cell nuclear antigen (PCNA) related factor, which acts as a regulator of DNA repair during DNA replication (8). Importantly, the interaction of PCNA-related factors with PCNA can increase the synthesis ability of DNA polymerase during the elongation of the leader strand, thereby accelerating the cell cycle process (9). The increase of cell proliferation in synovial fibroblasts in RA is related to the increase of PCNA level (10). Studies have found that the increased proliferation and decreased apoptosis of CD4+ T cells in RA synovium are also regulated by KIAA0101 gene expression (11). In this study, we found that KIAA0101 is highly expressed in RA synovial tissue, which further illustrates the correlation between KIAA0101 and RA. The protein encoded by FOXM1 is a transcriptional activator involved in cell proliferation. The encoded protein is phosphorylated in $\mathrm{M}$ phase and regulates the expression of several cell cycle genes, such as cyclin B1 and cyclin D1. The latest study found that the FOXM1/LINC00152 feedback loop regulates the proliferation and apoptosis of RA 
fibroblast-like synovial cells through the Wnt/ $\beta$-catenin signaling pathway (12). The FoxM1 inhibitor Streptococcus thiol inhibits the formation of human osteoclasts and may become a potential target for RA treatment (13). In this study, we found that FOXM1 is highly expressed in RA synovial tissue, which is consistent with previous research conclusions. The protein encoded by EGFR is a transmembrane glycoprotein, which is a member of the protein kinase superfamily. EGFR is a cell surface protein that binds to epidermal growth factor, thereby inducing receptor dimerization and tyrosine autophosphorylation, leading to cell proliferation. The expression of EGFR variant III (EGFRvIII) was detected in the synovial tissue and FLS (fibroblast-like synovial cells) of patients with aggressive RA. In FLS, EGFRvIll autophosphorylation destroys the proliferation, migration, invasion and anti-apoptosis ability of FLS expressing EGFRvIII (14). RA is associated with rs 17337023 polymorphism and elevated serum EGFR protein levels (15). In this study, we found that EGFR is low expressed in RA synovial lesions and is a potential biomarker in RA synovial lesions.

CDC20 seems to act as a regulatory protein, interacting with several other proteins at multiple points in the cell cycle. Previous studies have also found that CDC20 is a potential biomarker in RA synovitis (16), we found that $\mathrm{CDC} 20$ is highly expressed in RA synovitis, which shows that CDC20 plays an important role in RA synovial disease. TYMS has attracted people's interest as a target of cancer chemotherapeutic agents. It is considered to be the main site of action of 5-fluorouracil and some folate analogs. The polymorphism of this gene is not only related to the etiology of tumor formation, but also has a certain impact on the efficacy of methotrexate in RA patients (17). In this study, we found that TYMS is highly expressed in RA synovial lesions. RRM2 encodes one of the two different subunits of ribonucleotide reductase, which catalyzes the formation of deoxyribonucleotides from ribonucleotides. Studies have found that an enhanced RRM2 siRNA can be delivered to RA fibroblast-like synovial cells through liposome-protamine-DNA-siRNA complex and cell permeability peptide (18). In this study, we found that RRM2 is highly expressed in RA synovial lesions, which may be closely related to this transmission mechanism. JUN may be a transforming gene of avian sarcoma virus 17 , which encodes a protein that is highly similar to the viral protein and directly interacts with specific target DNA sequences to regulate gene expression. The latest study found that functional cfos/cjun mutants may change the transactivation ability of AP-1 complex, leading to local joint inflammation or destruction in RA patients (19). This study found that Jun is low in RA synovial tissue, which provides a new direction for the treatment of RA.

BUB1B encodes a kinase involved in spindle checkpoint function. The protein is located at the centromere and plays a role in the promotion of complex/circular body (APC/C) in the later stage of inhibition, delaying the occurrence of later stage and ensuring correct chromosome separation. TOP2A encodes DNA topoisomerase, an enzyme that controls and changes the topological state of DNA during the transcription process. This ribozyme is involved in processes such as chromosome condensation, chromatid separation, and torsional stress relief that occur during DNA transcription and replication. TOP2A is the target of many anticancer drugs, and many mutations of this gene are related to the development of drug resistance. The protein encoded by CCNA2 belongs to the highly conserved cyclin family, and its members play a role in regulating the cell cycle. This protein binds and activates cyclin- 
dependent kinase 2, thereby promoting the conversion of G1/S and G2/M. The correlation between BUB1B, TOP2A, CCNA2 and RA has not been reported so far. In this study, we found that BUB1B, TOP2A and CCNA2 are highly expressed in RA synovial lesions, and are potential biomarkers in the process of RA synovial lesions. Further research in synovial lesions is of great significance to the research on the mechanism and treatment of RA.

We also found two key miRNAs (miR-520d-5p and miR-139-5p). Previous studies have found that miR$520 \mathrm{~d}-5 p$ can promote the survival of human dermal fibroblasts exposed to a lethal dose of ultraviolet radiation (20). Undifferentiated collagen-bound miR-520d-5p has anti-tumor effect on undifferentiated cancer cells in a mouse xenograft model (21). Patients with Parkinson's disease increase serum miR$520 \mathrm{~d}-5 \mathrm{p}$ by regulating the expression of brain plasmin (22). In recent years, studies have found that miR$520 d-5 p$ can not only reduce the mutations of liver cancer cells and iPSC derivatives, but also play a role of tumor suppressor genes in cervical cancer by targeting PTK2 $(23,24)$. MiR-520d-5p also has a certain correlation with bone metabolism.,studies have found that miR-520d-5p regulates cartilage formation and chondrocyte metabolism by targeting HDAC1 (25). MiR-139 $5 \mathrm{p}$ is related to many diseases. Both endogenous and exogenous miR-139 5p can inhibit the development of clostridia-related colorectal cancer (26). Upregulation of miR-139-5p not only can reduce the oxidative stress and apoptosis of rat hippocampal neurons induced by epileptiform discharges by regulating the Notch pathway(27), but also can protect diabetic mice from liver tissue damage and oxidative stress by inhibiting the Notch signaling pathway(28). MiR-139-5p not only can negatively regulate PMP22 by targeting the NF-KB signaling pathway to inhibit gastric cancer cell proliferation (29), but also can regulate the proliferation, apoptosis and cell cycle of uterine leiomyoma cells by targeting TPD52 (30). However, the correlation between miR520d-5p and miR-139-5p and RA has not been reported. In this study, we found that miR-520d-5p and miR-139-5p are potential biomarkers in RA synovial lesions, Will be a new research direction.

We also obtained some immune cell related to RA synovial lesions. Compared with other immune activation markers, neutrophils are at the center of RA pathology (31) $₫$ The ratio of neutrophils to lymphocytes has great potential in predicting sustained remission of RA (32). Plasmacytoid dendritic cells participate in the local inflammatory environment of RA (33). B cells memory play an important role in the pathogenesis of RA. With the emergence of B cell targeted therapy, the regulation of B cells memory seems to be a key therapeutic target (34). The baseline expression of the B cell naive and $B$ cell memory marker FCRL 5 can predict the response of RA to rituximab (RTX) (35). The infiltration of inflammatory cells, especially the role of macrophages M1 that secrete a variety of inflammatory cytokines in RA cannot be ignored. In order to alleviate synovial inflammation, macrophages M1 must be eliminated or converted to an anti-inflammatory M2 phenotype. Folic acid modified silver nanoparticles (FA-AgNPs) can be actively introduced into macrophages $\mathrm{M} 1$, synergistically induce the reduction of macrophages $\mathrm{M} 1$ and the polarization of macrophages $M 2$, thereby effectively treating RA (36). Mast cells are immune cells that infiltrate the lubricating membrane. Their infiltration in the synovium of patients with early RA has been shown to be related to systemic inflammation, disease activity, and autoantibody positivity. Moreover, after using conventional synthetic disease-modifying anti-rheumatic drugs to treat early RA, mast cell-positive synovitis still exists (37). T cells Regulatory may participate in the process of RA bone 
destruction by mediating inflammatory immunity. The development of RA is related to the compartment defect of CD4+Foxp3+ T cells regulatory. Studies have found that the compartment defect of CD4+Foxp3+ T regulatory is negatively correlated with RA activity and antibody levels. Methotrexate treatment of early RA patients increased the proportion and absolute number of CD4+Foxp3+ $T$ regulatory cells with high levels of activation markers, suggesting that CD4+Foxp3+ T regulatory cells may be specific cell markers for successful RA treatment (1). Elevated serum IL-2 levels in patients with active RA are associated with abnormal levels of peripheral blood natural killer cells (38). Overexpression of IL-15 in RA can induce the proliferation and differentiation of natural killer cells. The expression of NKp46 and CD158e on natural killer cells may be a sign of the severity of RA (39). Although the relationship between these immune cells and RA has mostly been studied in the past, the role of dendritic cells resting, dendritic cells activated, mast cells resting and natural killer cells activated in RA synovial disease The role has not been clearly reported. In this study, we found that dendritic cells resting, dendritic cells activated, and mast cells resting are have high expression in RA, while natural killer cells activated have low expression in RA. It provides a new research idea for the pathogenesis of RA synovial lesions.

\section{Conclusion}

We downloaded GSE77298, GSE55457 and GSE55235 three datasets from GEO. After a series of bioinformatics analysis, we determined 10 Hub genes (KIAA0101, FOXM1, EGFR, CDC20, BUB1B, TYMS, TOP2A, RRM2, JUN and CCNA2) and two key miRNAs (miR-520d-5p and miR-139-5p). The correlation between BUB1B, TOP2A, CCNA2, two key miRNAs and RA have not been reported so far. We also identified some immune cells related to RA synovial lesions,the specific relevance between dendritic cells resting, dendritic cells activated, mast cells resting, natural killer cells activated and RA synovial lesions sexuality is rarely reported. These new findings provide a new research direction for the pathogenesis of RA synovial lesions. It is necessary to further verify and explore their potential value in the etiology and treatment of RA.

\section{Declarations}

Acknowledgements: We thank Mr Yongsong Cai for their support in bioinformatics and language.

Funding: This work was financially supported by the National Natural Science Foundation of China (No. 81772410, 81601877ه खChina Postdoctoral Science Foundation(No. 2020M673454)

Data availability claims: The data used in this article comes from Gene Expression Databases and has high availability.

Conflict of interest reputation: There is no conflict of interest between all authors of this article.

Authors' contributions reputation: Peng Xu and Mingyi Yang designed the study. Yani Su, Yao Ma Ke Xu and Ti Xia analyzed the microarray datasets and interpreted the results. Mingyi Yang downloaded the gene expression profile from the Gene Expression Omnibus. Mingyi Yang and Yani Su wrote and edited 
the manuscript. Peng Xu provided the foundation and support, All authors read and approved the final manuscript.

\section{References}

1. Avdeeva A, Rubtsov Y, Dyikanov D, et al. Regulatory T cells in patients with early untreated rheumatoid arthritis: Phenotypic changes in the course of methotrexate treatment. Biochimie. 2020; 174: 9-17.

2. Scott DL, Wolfe F. Rheumatoid arthritis. Lancet (London, England). 2010; 376: 1094-108.

3. Smolen JS, Aletaha D, McInnes IB. Rheumatoid arthritis. The Lancet. 2016; 388: 2023-38.

4. Xiong Y, Mi BB, Liu MF, et al. Bioinformatics Analysis and Identification of Genes and Molecular Pathways Involved in Synovial Inflammation in Rheumatoid Arthritis. Med Sci Monit. 2019; 25: 224656.

5. Huang DW, Sherman BT, Tan Q, et al. The DAVID Gene Functional Classification Tool: a novel biological module-centric algorithm to functionally analyze large gene lists. Genome Biol. 2007; 8: R183.

6. Ashburner M, Ball CA, Blake JA, et al. Gene ontology: tool for the unification of biology. The Gene Ontology Consortium. Nat Genet. 2000; 25: 25-9.

7. Yi XH, Zhang B, Fu YR, et al. STAT1 and its related molecules as potential biomarkers in Mycobacterium tuberculosis infection. J Cell Mol Med. 2020; 24: 2866-78.

8. Kais Z, Barsky SH, Mathsyaraja H, et al. KIAA0101 interacts with BRCA1 and regulates centrosome number. Mol Cancer Res. 2011; 9: 1091-9.

9. Burkovics P, Hajdu I, Szukacsov V, et al. Role of PCNA-dependent stimulation of 3'-phosphodiesterase and $3^{\prime}-5$ ' exonuclease activities of human Ape2 in repair of oxidative DNA damage. Nucleic Acids Res. 2009; 37: 4247-55.

10. Karouzakis E, Gay RE, Michel BA, et al. DNA hypomethylation in rheumatoid arthritis synovial fibroblasts. Arthritis and rheumatism. 2009; 60: 3613-22.

11. Aterido A, Palacio C, Marsal S, et al. Novel insights into the regulatory architecture of CD4+ T cells in rheumatoid arthritis. PLoS One. 2014; 9: e100690.

12. Wang W, Guo P, Chen M, et al. FOXM1/LINC00152 feedback loop regulates proliferation and apoptosis in rheumatoid arthritis fibroblast-like synoviocytes via Wnt/beta-catenin signaling pathway. Biosci Rep. 2020; 40.

13. Hasegawa T, Kikuta J, Sudo T, et al. Identification of a novel arthritis-associated osteoclast precursor macrophage regulated by FoxM1. Nat Immunol. 2019; 20: 1631-43.

14. Niu J, Li C, Jin Y, et al. Identification and suppression of epidermal growth factor receptor variant III signaling in fibroblast-like synoviocytes from aggressive rheumatoid arthritis by the mimotope. Immunol Lett. 2018; 198: 74-80. 
15. Huang CM, Chen HH, Chen DC, et al. Rheumatoid arthritis is associated with rs 17337023 polymorphism and increased serum level of the EGFR protein. PLoS One. 2017; 12: e0180604.

16. Li Z, Xu M, Li R, et al. Identification of biomarkers associated with synovitis in rheumatoid arthritis by bioinformatics analyses. Biosci Rep. 2020; 40.

17. Muralidharan N, Misra DP, Jain VK, et al. Effect of thymidylate synthase (TYMS) gene polymorphisms with methotrexate treatment outcome in south Indian Tamil patients with rheumatoid arthritis. Clin Rheumatol. 2017; 36: 1253-59.

18. WANG X, WANG X, SUN J, et al. An enhanced RRM2 siRNA delivery to rheumatoid arthritis fibroblastlike synoviocytes through a liposome-protamine-DNA-siRNA complex with cell permeable peptides. INTERNATIONAL JOURNAL OF MOLECULAR MEdICINE. 2018; 42: 2393-402.

19. Huber R, Augsten S, Kirsten H, et al. Identification of New, Functionally Relevant Mutations in the Coding Regions of the Human Fos and Jun Proto-Oncogenes in Rheumatoid Arthritis Synovial Tissue. Life (Basel). 2020; 11.

20. Ishihara $Y$, Tsuno S, Ping B, et al. Hsa-miR-520d-5p promotes survival in human dermal fibroblasts exposed to a lethal dose of UV irradiation. NPJ Aging Mech Dis. 2016; 2: 16029.

21. Ishihara Y, Tsuno S, Kuwamoto S, et al. Correction to: Tumor-suppressive effects of atelocollagenconjugated hsa-miR-520d-5p on un-differentiated cancer cells in a mouse xenograft model. BMC Cancer. 2017; 17: 666.

22. Jin L, Wan W, Wang L, et al. Elevated microRNA-520d-5p in the serum of patients with Parkinson's disease, possibly through regulation of cereloplasmin expression. Neurosci Lett. 2018; 687: 88-93.

23. Zhang L, Liu F, Fu Y, et al. MiR-520d-5p functions as a tumor-suppressor gene in cervical cancer through targeting PTK2. Life Sci. 2020; 254: 117558.

24. Miura N, Ishihara Y, Miura Y, et al. miR-520d-5p can reduce the mutations in hepatoma cancer cells and iPSCs-derivatives. BMC Cancer. 2019; 19: 587.

25. Lu1 J, Zhou1 Z, Sun B. MiR-520d-5p modulates chondrogenesis and chondrocyte metabolism through targeting HDAC1. Aging (Albany NY). 2020; 12: 18545-60.

26. Zhao Y, Tao Q, Li S, et al. Both endogenous and exogenous miR-139-5p inhibit Fusobacterium nucleatum-related colorectal cancer development. Eur J Pharmacol. 2020; 888: 173459.

27. Zhao C, Yang F, Wei X, et al. MiR-139-5p Upregulation Alleviated Spontaneous Recurrent Epileptiform Discharge-induced Oxidative Stress and Apoptosis in Rat Hippocampal Neurons via Regulating the Notch Pathway. Cell Biol Int. 2020.

28. Wei H, Huang L, Wei F, et al. Up-regulation of miR-139-5p protects diabetic mice from liver tissue damage and oxidative stress through inhibiting Notch signaling pathway. Acta Biochim Biophys Sin (Shanghai). 2020; 52: 390-400.

29. Hou J, Zhuo H, Chen X, et al. MiR-139-5p negatively regulates PMP22 to repress cell proliferation by targeting the NF-kappaB signaling pathway in gastric cancer. Int J Biol Sci. 2020; 16: 1218-29. 
30. H C, H X, YG M, et al. miR-139-5p Regulates Proliferation, Apoptosis, and Cell Cycle of Uterine Leiomyoma Cells by Targeting TPD52 [Expression of Concern]. Onco Targets Ther. 2020; 13: 6855.

31. Birkelund S, Bennike TB, Kastaniegaard K, et al. Proteomic analysis of synovial fluid from rheumatic arthritis and spondyloarthritis patients. Clin Proteomics. 2020; 17: 29.

32. Chandrashekara S, Lingaraju DC, Renuka P, et al. Potential of neutrophil to lymphocyte ratio in predicting sustained remission in rheumatoid arthritis compared to other immune activation markers. Indian J Med Res. 2020; 152: 234-43.

33. Cavanagh LL, Boyce A, Smith L, et al. Rheumatoid arthritis synovium contains plasmacytoid dendritic cells. Arthritis Res Ther. 2005; 7: R230-40.

34. Mahmood Z, Schmalzing M, Dorner T, et al. Therapeutic Cytokine Inhibition Modulates Activation and Homing Receptors of Peripheral Memory B Cell Subsets in Rheumatoid Arthritis Patients. Front Immunol. 2020; 11: 572475.

35. Owczarczyk K, Cascino MD, Holweg C, et al. Fc receptor-like 5 and anti-CD20 treatment response in granulomatosis with polyangiitis and microscopic polyangiitis. JCI Insight. 2020; 5.

36. Yang Y, Guo L, Wang Z, et al. Targeted silver nanoparticles for rheumatoid arthritis therapy via macrophage apoptosis and Re-polarization. Biomaterials. 2021; 264: 120390.

37. Rivellese F, Rossi FW, Giorli G, et al. Persistence of Mast Cell-Positive Synovitis in Early Rheumatoid Arthritis Following Treatment With Conventional Synthetic Disease Modifying Anti-Rheumatic Drugs. Front Pharmacol. 2020; 11: 1051.

38. Li B, Guo Q, Wang Y, et al. Increased Serum Interleukin-2 Levels Are Associated with Abnormal Peripheral Blood Natural Killer Cell Levels in Patients with Active Rheumatoid Arthritis. Mediators Inflamm. 2020; 2020: 6108342.

39. Lin SJ, Hsu CY, Kuo ML, et al. Phenotypic and functional characterization of natural killer cells in rheumatoid arthritis-regulation with interleukin-15. Sci Rep. 2020; 10: 5858.

\section{Tables}

Table1®Hub gene. 


\begin{tabular}{|c|c|c|c|}
\hline Gene & Details & Frequency & Species \\
\hline KIAA0101 & KIAA0101(KIAA0101) & 9 & $\begin{array}{l}\text { Homo } \\
\text { sapiens }\end{array}$ \\
\hline FOXM1 & forkhead box M1(FOXM1) & 9 & $\begin{array}{l}\text { Homo } \\
\text { sapiens }\end{array}$ \\
\hline EGFR & epidermal growth factor receptor(EGFR) & 9 & $\begin{array}{l}\text { Homo } \\
\text { sapiens }\end{array}$ \\
\hline CDC20 & cell division cycle $20(\mathrm{CDC} 20)$ & 10 & $\begin{array}{l}\text { Homo } \\
\text { sapiens }\end{array}$ \\
\hline BUB1B & $\begin{array}{l}\text { BUB1 mitotic checkpoint serine/threonine kinase } \\
\text { B(BUB1B) }\end{array}$ & 10 & $\begin{array}{l}\text { Homo } \\
\text { sapiens }\end{array}$ \\
\hline TYMS & thymidylate synthetase(TYMS) & 11 & $\begin{array}{l}\text { Homo } \\
\text { sapiens }\end{array}$ \\
\hline TOP2A & topoisomerase (DNA) II alpha(TOP2A) & 11 & $\begin{array}{l}\text { Homo } \\
\text { sapiens }\end{array}$ \\
\hline RRM2 & ribonucleotide reductase regulatory subunit M2(RRM2) & 12 & $\begin{array}{l}\text { Homo } \\
\text { sapiens }\end{array}$ \\
\hline JUN & $\begin{array}{l}\text { Jun proto-oncogene, AP-1 transcription factor } \\
\text { subunit(JUN) }\end{array}$ & 12 & $\begin{array}{l}\text { Homo } \\
\text { sapiens }\end{array}$ \\
\hline CCNA2 & cyclin A2(CCNA2) & 13 & $\begin{array}{l}\text { Homo } \\
\text { sapiens }\end{array}$ \\
\hline
\end{tabular}

\section{Figures}



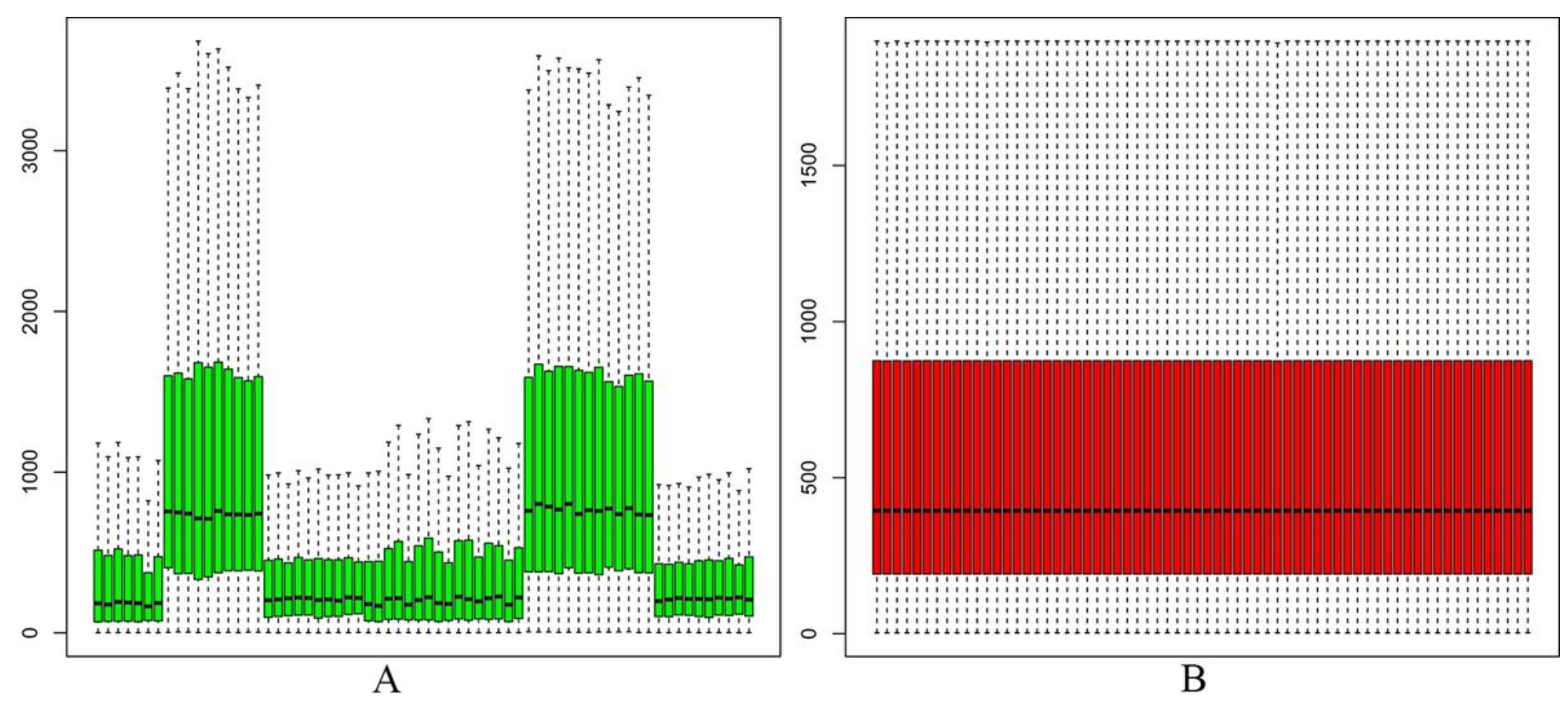

Figure 1

GSE77298, GSE55457 and GSE55235 three data sets for batch calibration. A: Before batch calibration; B: After batch calibration.

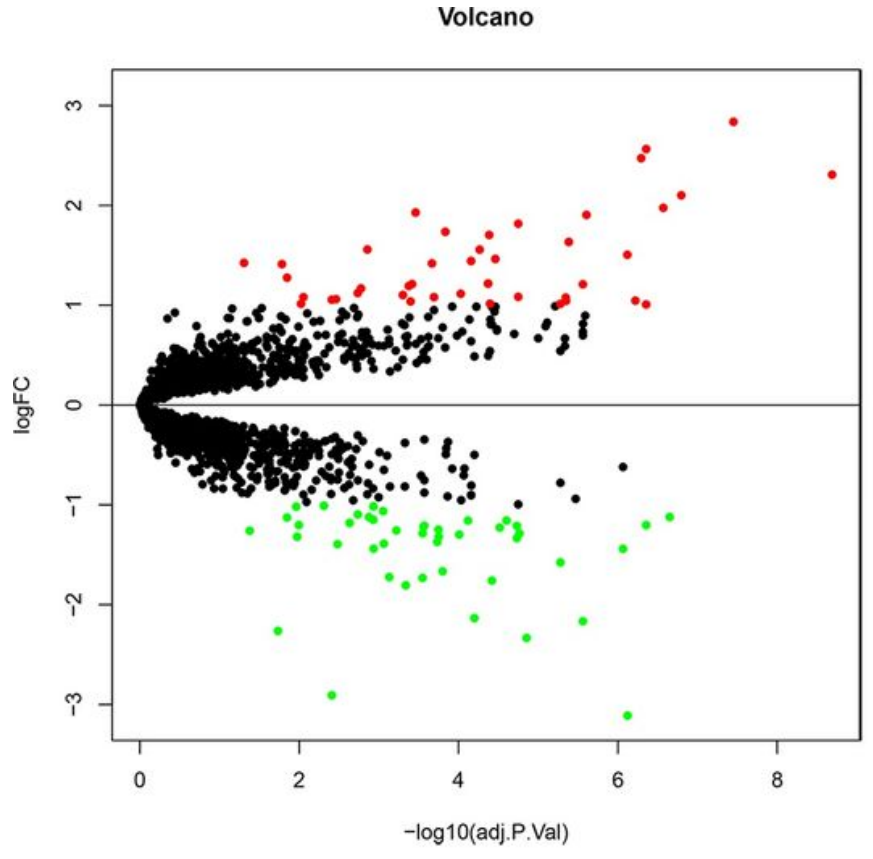

A

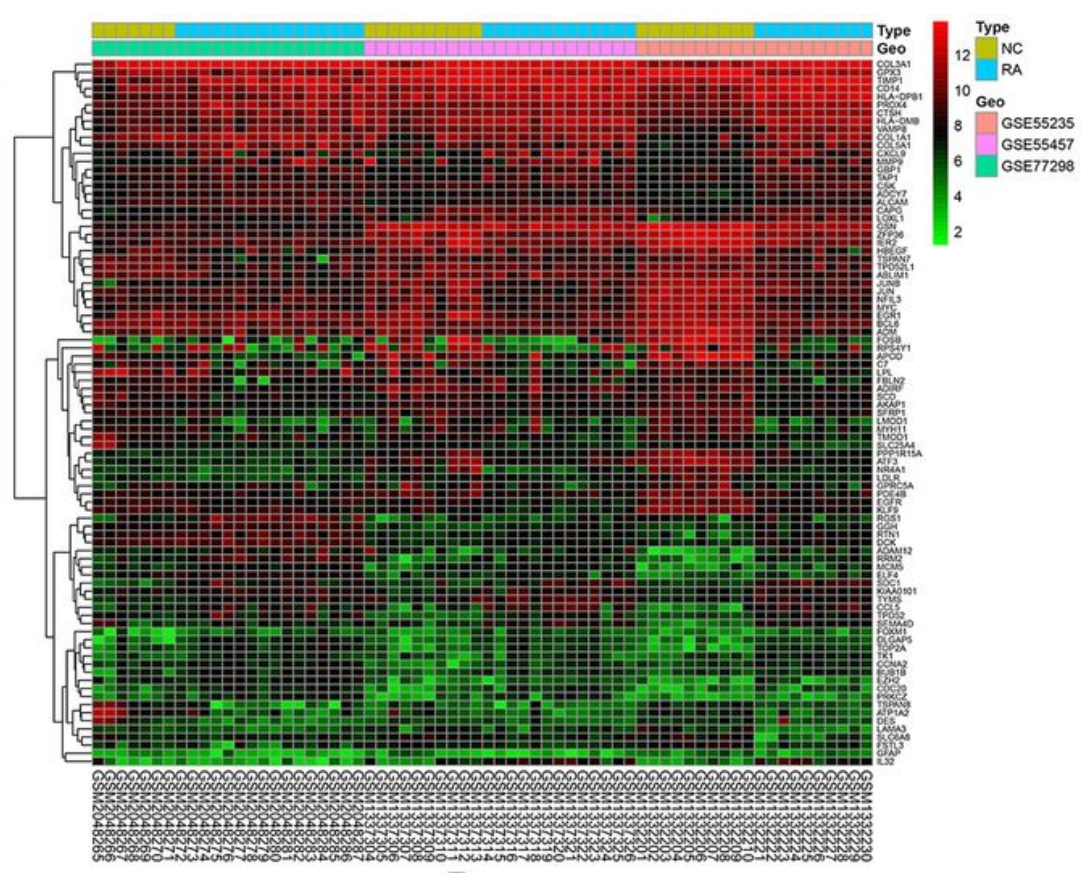

B

Figure 2

Differentially expressed genes identified in the three data sets GSE77298, GSE55457 and GSE55235. A: Volcano map of differentially expressed genes. Black is a gene with no difference, red is an up-regulated gene, and green is a down-regulated gene. B: Heat map of differentially expressed genes. The abscissa is the samples of the three data sets, the ordinate is the differentially expressed genes, red is high expression, and green is low expression. 

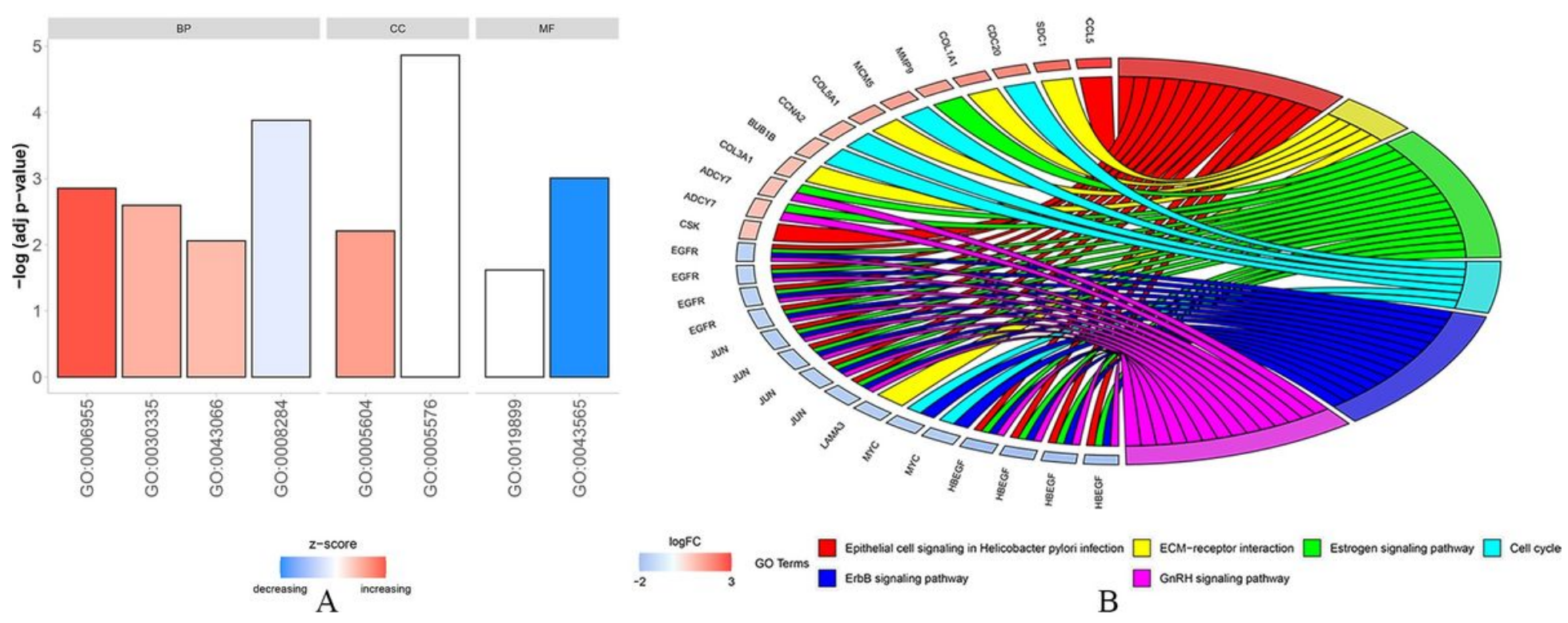

Figure 3

Enrichment analysis of differentially expressed genes. A: GO enrichment, z-score=(number of upregulated genes-number of down-regulated genes)/total number of genes. The bluer the color, the smaller the $z$-score, indicating that the more down-regulated genes are enriched in this term. The redder the color, the higher the z-score, which means that the more up-regulated genes are enriched in this term. B: KEGG enrichment. Different colors represent different pathways. The bluer the color of differentially expressed genes, the smaller the logFC value, and the redder the color of differentially expressed genes, the greater the $\log F C$ value.

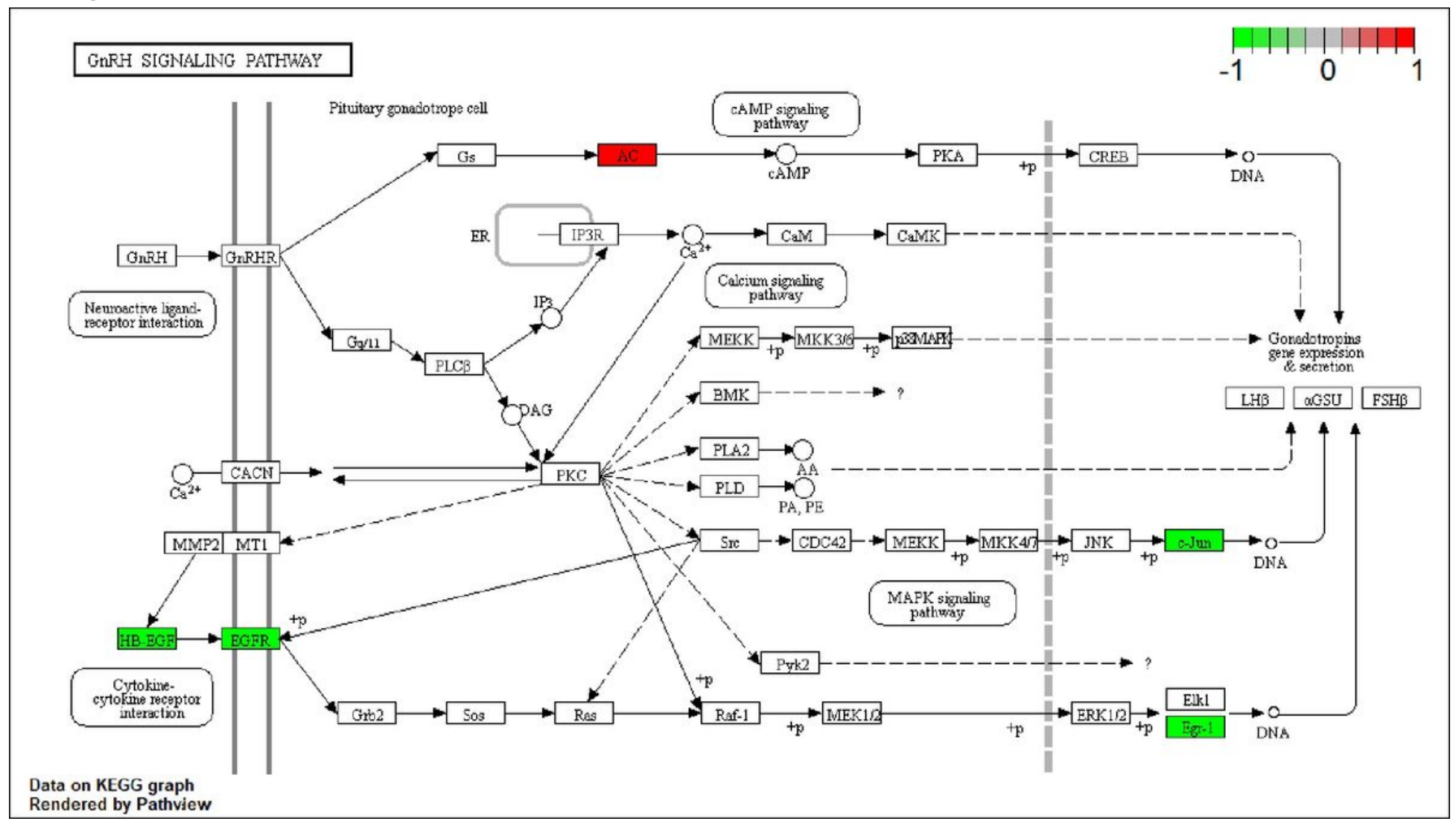


Figure 4

GnRH signal pathway diagram of the enrichment analysis results of differentially expressed genes KEGG.

KIAA0101

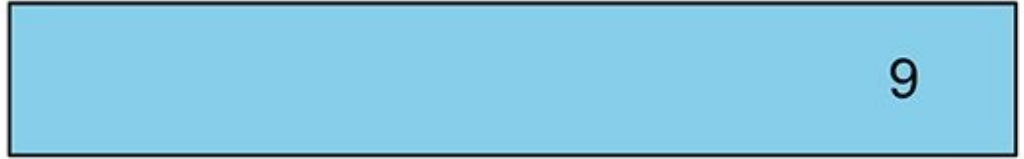

FOXM1

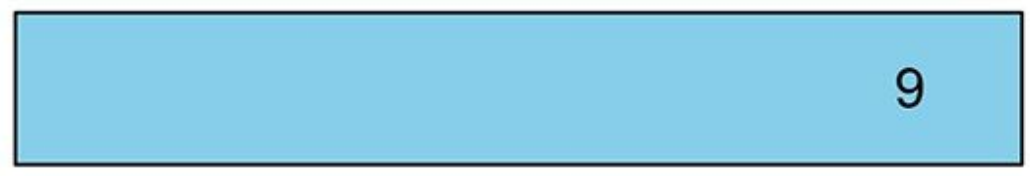

EGFR

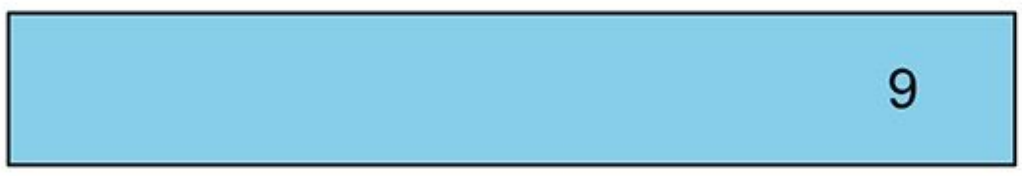

CDC20

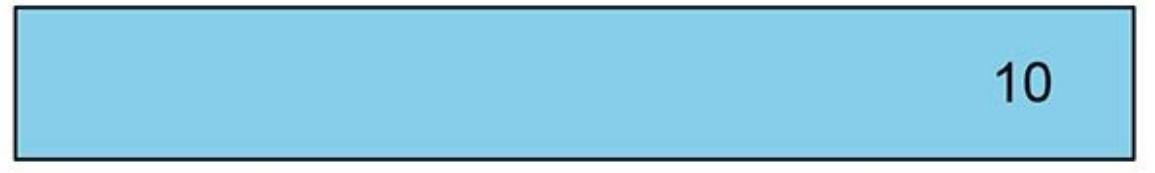

BUB1B

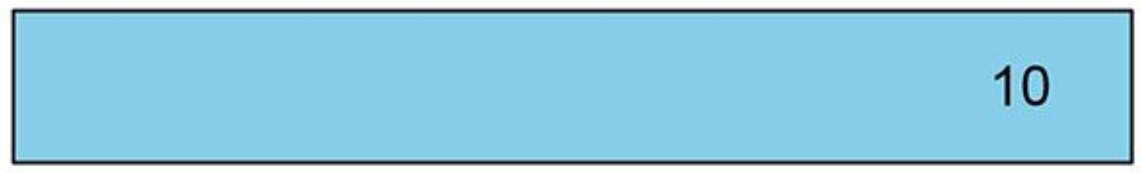

TYMS

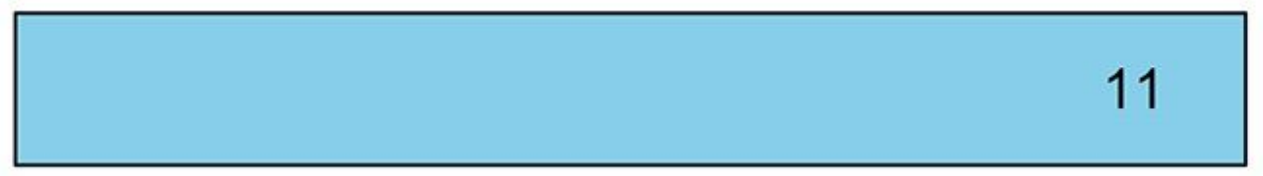

TOP2A

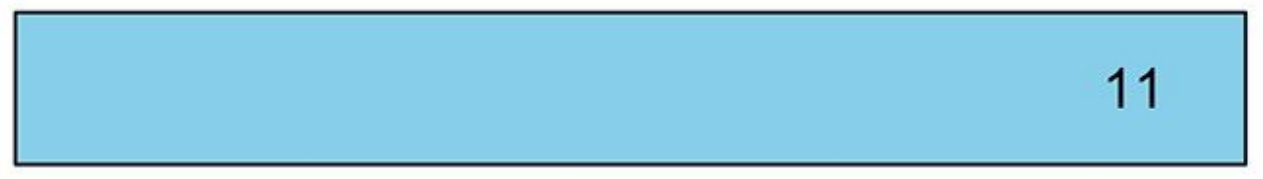

RRM2

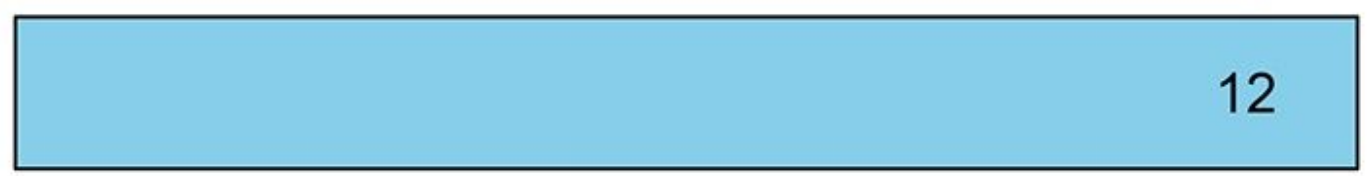

JUN

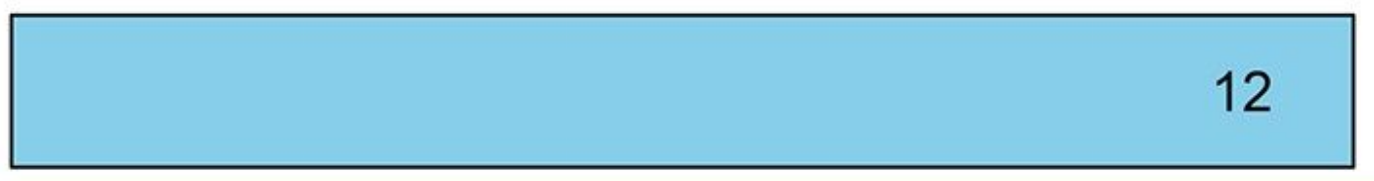

CCNA2
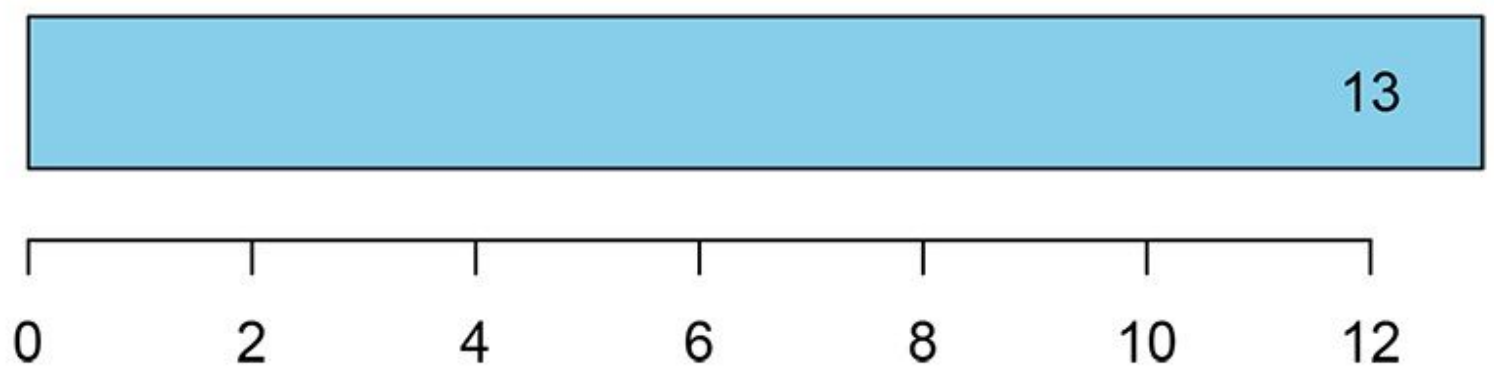

Figure 5

10 Hub genes, the left is the gene name, and the number on the right is the number of times the gene appears in the PPI network. 


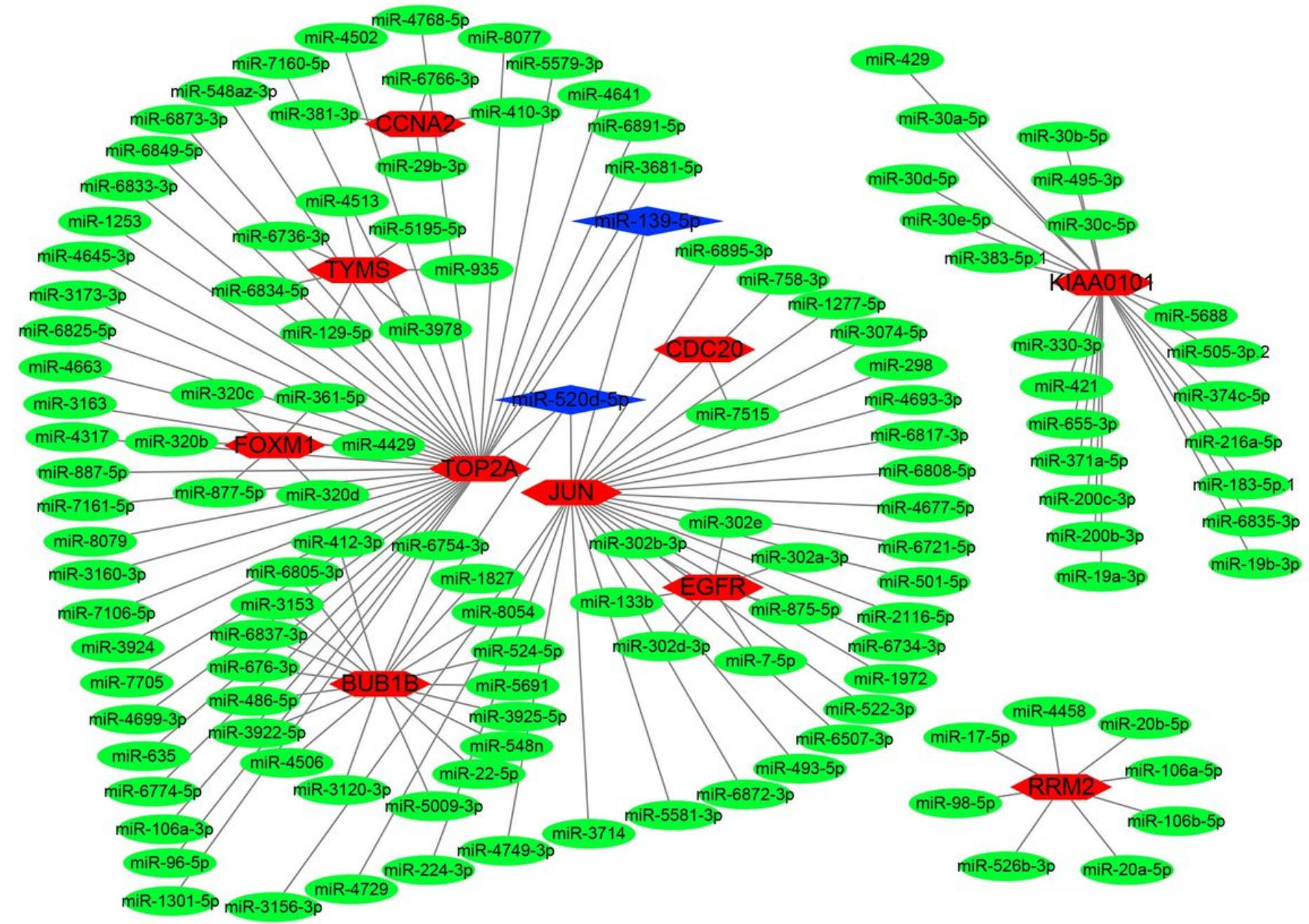

\section{Figure 6}

The interaction network between the Hub gene and its targeted miRNAs. Red is the Hub gene, green is the targeted miRNAs, and blue is the miRNAs that target two or more Hub genes. 


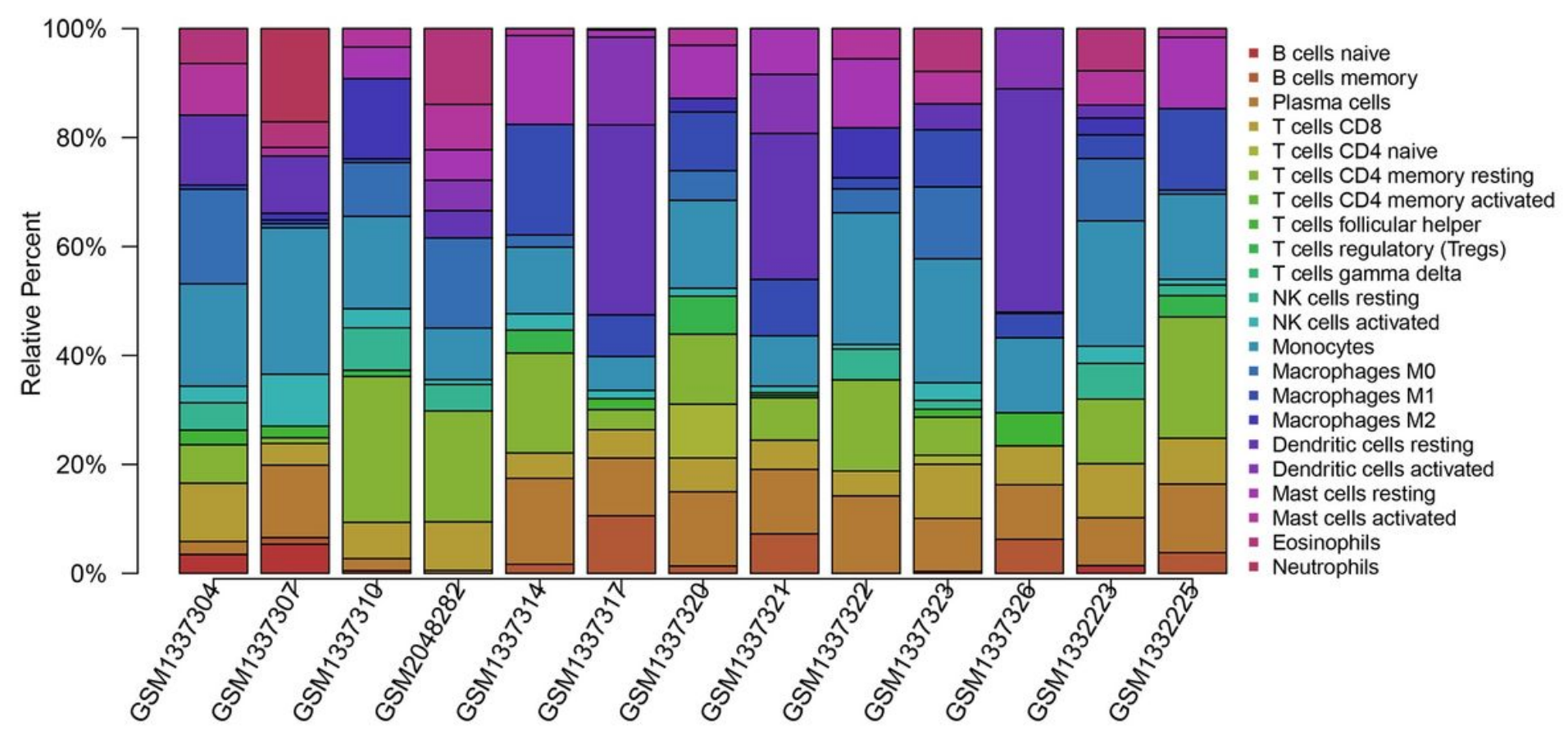

Figure 7

Immune cell matrices for the three data sets. The abscissa is the sample with $\mathrm{P}<0.05$, and the ordinate is 22 kinds of immune cells. Different colors represent different immune cells. The total of 22 immune cells in each sample is 1 .

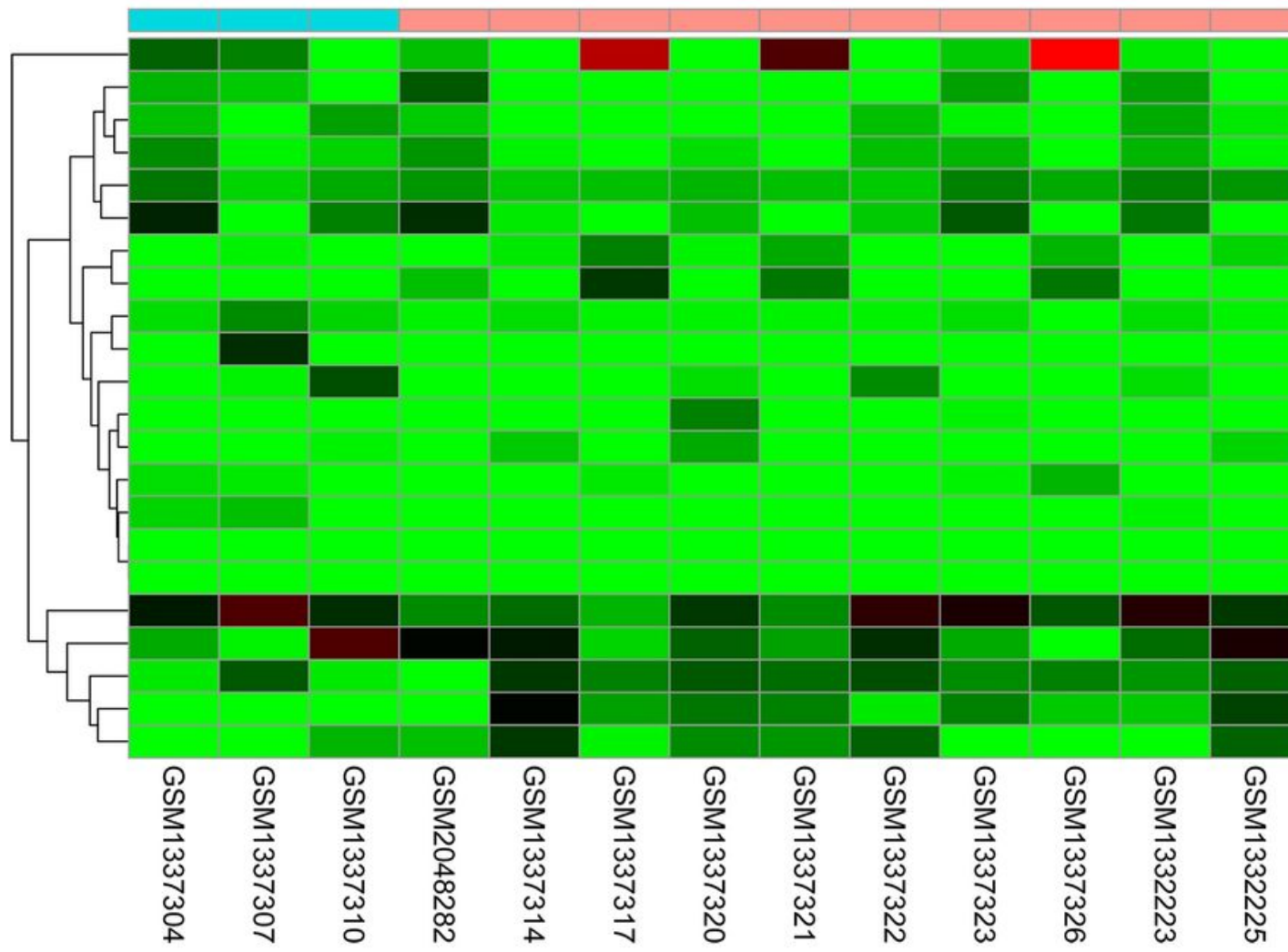

Type

Dendritic cells resting

Eosinophils

NK cells resting

Mast cells activated

T cells CD8

Macrophages MO

$B$ cells memory

Dendritic cells activated

NK cells activated

Neutrophils

Macrophages M2

$\mathrm{T}$ cells $\mathrm{CD} 4$ naive

$T$ cells regulatory (Tregs)

$T$ cells follicular helper

$B$ cells naive

T cells CD4 memory activated

$T$ cells gamma delta

Monocytes

T cells CD4 memory resting

Plasma cells

Macrophages M1

Mast cells resting 
Immune cell matrix heat maps of the three data sets. The abscissa is the sample with $\mathrm{P}<0.05$, and the ordinate is 22 kinds of immune cells. The upper blue is the normal synovial tissue sample, and the red is the RA synovial tissue sample. The redder the color, the higher the expression, and the greener the color, the lower the expression.

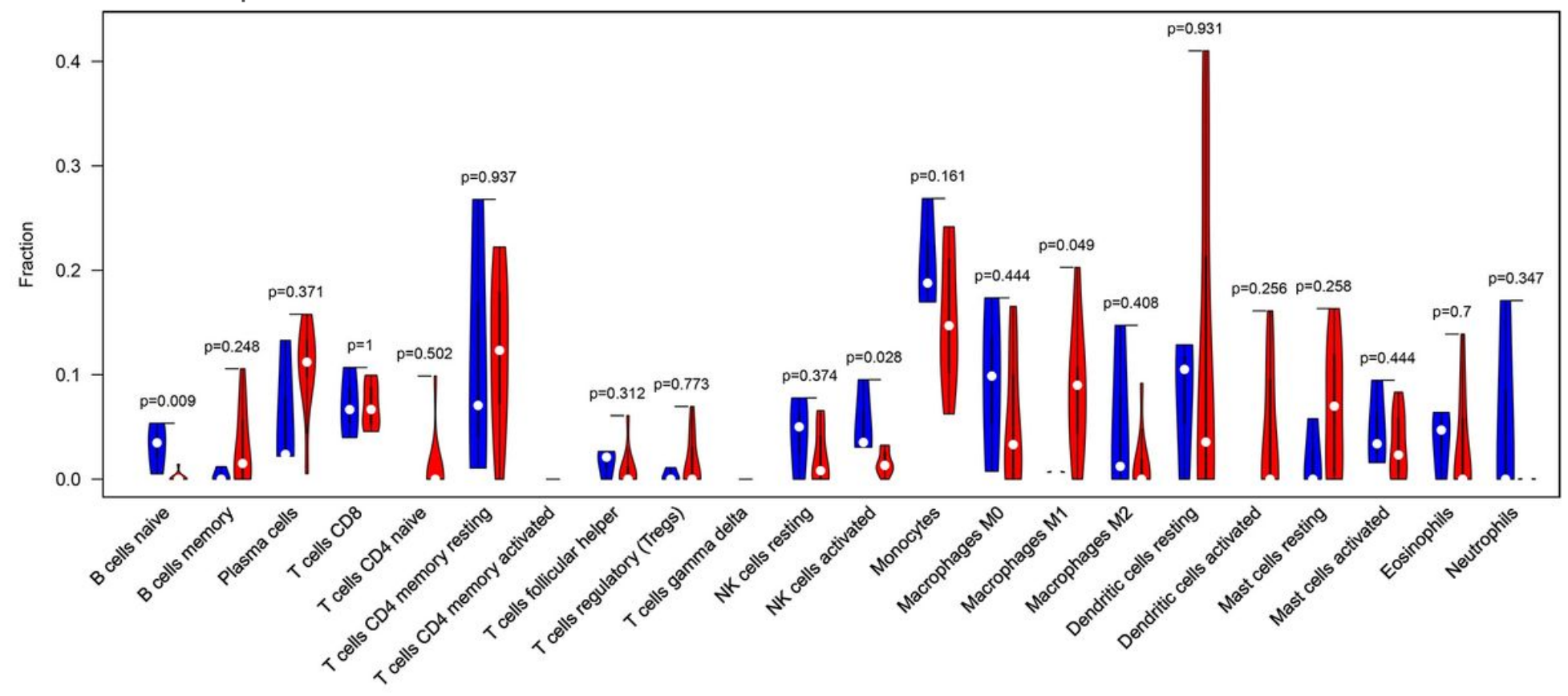

Figure 9

Violin image of immune cells in 22 normal synovial tissue and RA synovial tissue. Blue represents normal synovial tissue and red represents RA synovial tissue. 